\title{
Nuevos registros de Holochilus chacarius (Rodentia, Cricetidae) en la cuenca del río Carcarañá (Santa Fé, Argentina) y consideraciones biogeográficas
}

\author{
New records of Holochilus chacarius (Rodentia, Cricetidae) in the Carcarañá river basin \\ (Santa Fé province, Argentina) and biogeographical considerations
}

Pablo G. Rimoldi ${ }^{1,2}$

https://orcid.org/0000-0002-3717-7649

primoldi04@gmail.com

Nicolás R. Chimento* 3,4

https://orcid.org/0000-0002-6340-4358

nicochimento@hotmail.com

\section{* Corresponding author}

1 Zoología general, Facultad de Ciencias Agrarias, Universidad Nacional de Rosario, Argentina.

2 Biología y Ecología, Facultad de Ciencias Veterinarias, Universidad Nacional de Rosario, Argentina.

3 Laboratorio de Anatomía Comparada y Evolución de los Vertebrados, Museo Argentino de Ciencias Naturales "Bernardino Rivadavia", Argentina.

4 Consejo Nacional de Investigaciones Científicas y Técnicas (CONICET), Argentina.

\section{Citación}

Rimoldi PG, Chimento NR. 2021. Nuevos registros de Holochilus chacarius (Rodentia, Cricetidae) en la cuenca del río Carcarañá (Santa Fé, Argentina) y consideraciones biogeográficas. Revista peruana de biología 28(4): e21007001- 008 (Noviembre 2021). doi: http://dx.doi.org/10.15381/rpb.v28i4.21007

$\begin{array}{ll}\text { Presentado: } & 11 / 08 / 2021 \\ \text { Aceptado: } & 03 / 11 / 2021 \\ \text { Publicado online: } & 26 / 11 / 2021 \\ \text { Editor: } & \text { Victor Pacheco }\end{array}$

\section{Resumen}

La recolección y análisis de egagrópilas resulta un método adecuado en el estudio de presas pequeñas, debido a que permite el estudio de áreas extensas, genera gran cantidad y variedad de individuos por lote. El orden Strigiformes cuenta con el mayor número de estudios de dieta de aves rapaces a nivel mundial, destacándose los referidos a Tyto furcata con gran cantidad de contribuciones sobre el análisis de sus regurgitados. El estudio de las regurgitaciones de esta especie ha ayudado a realizar nuevas interpretaciones sobre la distribución, taxonomía y conservación de muchas especies de roedores. Aquí, se describe la presencia de Holochilus chacarius en el sur de la provincia de Santa Fe a partir de la recolección y análisis de egagrópilas de la lechuza del campanario (Tyto furcata). Estos registros amplían la distribución de $H$. chacarius en la provincia y contribuyen a actualizar los mapas de distribución existentes.

\section{Abstract}

The collection and analysis of pellets is an adequate method in the study of small prey, because it allows the study of large areas, generates a large number and variety of individuals per lot. The order Strigiformes has the largest number of studies on the diet of birds of prey worldwide, highlighting those referring to Tyto furcata with a large number of contributions on the analysis of its regurgitates. The study of the regurgitations of this species has helped to make new interpretations about the distribution, taxonomy, and conservation of many species of rodents. Here, the presence of Holochilus chacarius is described in the south of the province of Santa Fe from the collection and analysis of pellets of the Barn Owl (Tyto furcata). These records expand the distribution of $\mathrm{H}$. chacarius in the province and contribute to updating the existing distribution maps.

Palabras clave:

Holochilus chacarius; Tyto furcada; distribución; Santa Fe.

Keywords:

Holochilus chacarius; Tyto furcada; distribution; Santa Fe. 


\section{Introducción}

El estudio de la dieta de las aves rapaces a partir del análisis de sus egagrópilas puede ayudar a conocer mejor la distribución, abundancia, conducta y vulnerabilidad de las especies presa (Fulk 1976, Marti 1987). Además, en algunos casos ha permitido el descubrimiento de nuevas especies de roedores (e.g., Massoia 1979) y la extensión de la distribución conocida de otras (e.g., Pardiñas \& Galliari 1998, Podestá et al. 2000, Teta et al. 2006a, Teta \& Pardiñas 2007, Udrizar Sauthier \& Carrera 2013).

Las egagrópilas son aglomerados de restos de presas no digeridos, como huesos, pelos, plumas, exoesqueletos de artrópodos, entre otros, consumidas por aves y regurgitadas periódicamente, en general en sitios de descanso y anidación (Errington 1930, Andrews 1990). La recolección y estudio de éstas, resulta un método adecuado en el estudio de presas pequeñas, debido a que permite el estudio de áreas extensas, genera gran cantidad y variedad de individuos por lote. En varias contribuciones, se ha considerado que el número y frecuencia de presas halladas en las egagrópilas son suficientemente representativas de la disponibilidad de presas en una determinada área (Errington 1930, Bonvicino \& Bezerra 2003, Millán de la Peña et al. 2003, Pardiñas et al. 2003, Trejo \& Lambertucci 2007, Gonzalez-Fischer et al. 2012, Lyman 2012).

El orden Strigiformes cuenta con el mayor número de estudios de dieta de aves rapaces a nivel mundial (Bó et al. 2007). En esta rama de la ecología, los estudios tróficos en aves rapaces son realizados comúnmente para la familia Tytonidae, y se destaca a la lechuza de campanario (Tyto furcata) por estar entre las especies con mayor número de contribuciones sobre el análisis de sus regurgitados (Gonzáles-Calderón 2017).

Tyto furcata es una especie que habita en gran variedad de ambientes, incluso muy antropizados (Tylor 2004). El estudio de las regurgitaciones de esta especie ha ayudado a realizar nuevas interpretaciones sobre la distribución, taxonomía y conservación de muchas especies de roedores (Massa et al. 2014a). Por ejemplo, se ha registrado que la variación de la densidad de una especie presa en ecosistemas templados puede correlacionarse con la proporción de esa especie presa en la dieta de Tyto furcata, e incluso dependiendo de la densidad de otras especies presentes en el área (Bernard et al. 2010). En general, estos mamíferos constituyen su principal fuente de alimentos, aunque también se alimentan de pequeños marsupiales y murciélagos (Massoia 1988, Massa et al. 2014 b, Idoeta et al. 2020), y en menor proporción de pequeñas aves, anuros y reptiles (Massoia \& Fornes 1964).

Holochilus chacarius es una especie de roedor cricétido, que muestra una distribución por la zona chaqueña de Argentina, Paraguay, Bolivia y parte del Cerrado de Brasil, llegando hasta el delta inferior de la desembocadura de los ríos Paraná y Uruguay (Massa et al. 2019, Prado et al. 2021). Esta especie no presenta registros en las cuencas fluviales del interior de las provincias de Buenos Aires y Santa Fe, donde sí aparece H. vulpinus (Torres \& Massa 2019).
En este trabajo se utiliza el análisis de dieta de Tyto furcata como método indirecto, pero con alto grado de fiabilidad, para establecer la presencia de Holochilus chacarius en distintas localidades del sur y oeste de la provincia de Santa Fe. El objetivo de la presente contribución es presentar nuevos registros de $H$. chacarius para la provincia de Santa Fe, en un área donde previamente no había sido registrado, y discutir las posibles hipótesis acerca de la presencia de ésta y la ausencia de $H$. vulpinus en los muestreos.

\section{Materiales y métodos}

Área de estudio. - La provincia de Santa Fe, Argentina, se ubica en la región del centro-este del país, en una zona de clima templado con estacionalidad marcada, observando variaciones relacionadas a su extensión territorial con predominio de su eje norte-sur, que determina que en el norte el clima se aproxima más a un perfil subtropical, mientras que al sur es más bien de tipo templado-frío (Lewis \& Collantes 1974, Coronel \& Sacchi 2006).

La zona de estudio se ubica en el tercio más austral de su territorio con precipitaciones que oscilan entre los 800 y $1000 \mathrm{~mm}$ anuales, ubicándose entre las isotermas anuales de entre los 16 y $18{ }^{\circ} \mathrm{C}$, variables entre días con heladas en época invernal y temperaturas estivales que pueden alcanzar más de $35^{\circ} \mathrm{C}$ (Coronel \& Sacchi 2006). Se corresponde con el bioma denominado "pampa húmeda argentina", caracterizado por ser una extensa planicie con escasa pendiente que, originalmente fue un ecosistema de pastizal prácticamente continuo, sin especies leñosas excepto en algunos corredores, asociados principalmente a los cuerpos de agua de tipo lótico (ríos y arroyos) (Cabrera 1976).

En la actualidad presenta un grado de subdivisión y utilización del suelo que modificó su aspecto original, convirtiéndose en una planicie donde predominan los cultivos de granos y oleaginosas. En general, se puede considerar a la zona de estudio como un área de alta producción agrícola, con un crecimiento en detrimento de la ganadera y en una sobreexplotación del suelo, con prácticas intensivas de dobles cultivos anuales, como por ejemplo trigo y soja. Esto ha provocado una disminución importante de los ambientes naturales, un cambio en la estructura y funcionamiento de los ecosistemas y en la mayoría de los casos su fragmentación (Martínez 2010, Bilenca et al. 2012).

Para esta investigación se establecieron distintos puntos de muestreo (a partir de la presencia de perchas activas de Tyto furcata) en siete localidades del sur de Santa Fe. De esta manera quedó establecido para el departamento Caseros las localidades de Casilda, Los Molinos, Arequito, Los Nogales y Los Quirquinchos; para el departamento Iriondo, la localidad de Totoras; y para el departamento Belgrano, la localidad Montes de Oca.

A fin de establecer asociaciones entre los registros de presencia y el tipo de ambiente se estableció una categorización según uso del suelo siguiendo a Rimoldi y Curti (2021), el cual quedó establecido de la siguiente mane- 
ra: Ambiente Urbano (U), donde hay dominancia de viviendas humanas, y los espacios verdes se reducen a los patios de las mismas y a las veredas donde se disponen árboles y arbustos mayoritariamente exóticos; Ambiente Rural Antropizados (RA), donde la producción agrícola crece en detrimento de la ganadería con una sobreexplotación del suelo con prácticas intensivas de dobles cultivos anuales, como por ejemplo maíz y soja; y Ambiente Rural No Antropizado o Seminatural (RNAN), donde las limitantes edáficas o geomorfológicas permiten la presencia de formaciones vegetales autóctonas sin agricultura o cría intensiva de ganado.

Colecta de datos. - Entre enero y octubre de 2020 se colectaron egagrópilas mensualmente en puntos previamente establecidos en los sitios de muestreo. En todos los casos se recogió el total de estas dejando la percha limpia. Tratándose de ámbitos restringidos, resultó relativamente sencillo rastrear en toda la superficie expuesta bajo las perchas, obteniéndose todo el material disponible.

Las egagrópilas se colocaron en bolsas de papel rotuladas (con el nombre del ambiente relevado, coordenadas, fecha y cantidad) y luego en bolsas de polietileno herméticamente selladas. Se respetaron todas las medidas de bioseguridad (uso de guantes de látex y barbijo en la colecta) hasta el secado del material, el cual se realizó en laboratorio con una estufa, a $70{ }^{\circ} \mathrm{C}$ durante $48 \mathrm{~h}$ (Muñoz-Pedreros \& Yañez 2004). Posteriormente fueron procesadas, utilizando instrumental quirúrgico para extraer los restos de mandíbulas y cráneos de los animales encontrados. Aquellas egagrópilas que estaban compactadas fueron remojadas en agua durante tres horas para realizar la separación.

Las mandíbulas y cráneos de las presas capturadas se compararon con muestras identificadas en distintas colecciones osteológicas y literatura especializada (Voglino et al. 2004, Gómez Villafañe et al. 2005, Courtalon et al. 2013, Patton et al. 2015, Brandao \& Nascimento 2015). Se consideró como un individuo a los pares de mandíbulas de la misma especie y/o cráneo.

Los materiales estudiados están depositados en la Colección de Zoología General de la Facultad de Ciencias Agrarias, Universidad Nacional de Rosario.

\section{Resultados}

Se obtuvo un total de 6236 individuos recuperados de 2598 egagrópilas. Del total, el 96\% pertenece a micromamíferos, mientras que el 4\% restante corresponde a ejemplares de anfibios, reptiles y aves. El 91\% ( $n=5448)$ del total de micromamíferos recuperados, correspondieron a roedores nativos de la familia Cricetidae, seguida por los roedores introducidos de la familia Muridae $n$ = 476 (7.96\%). La menor proporción de presas estuvo representada por las familias Caviidae $n=45(0.76 \%)$, Molossidae $n=6(0.1 \%)$ y Leporidae $(0.016 \%)$.

A partir de los resultados obtenidos se pudo establecer la presencia de 31 individuos de Holochilus chacarius brindando de esta forma los primeros registros de esta especie para los departamentos Caseros, Iriondo y Belgrano (provincia de Santa Fe, Tabla 1 y Figura 1).
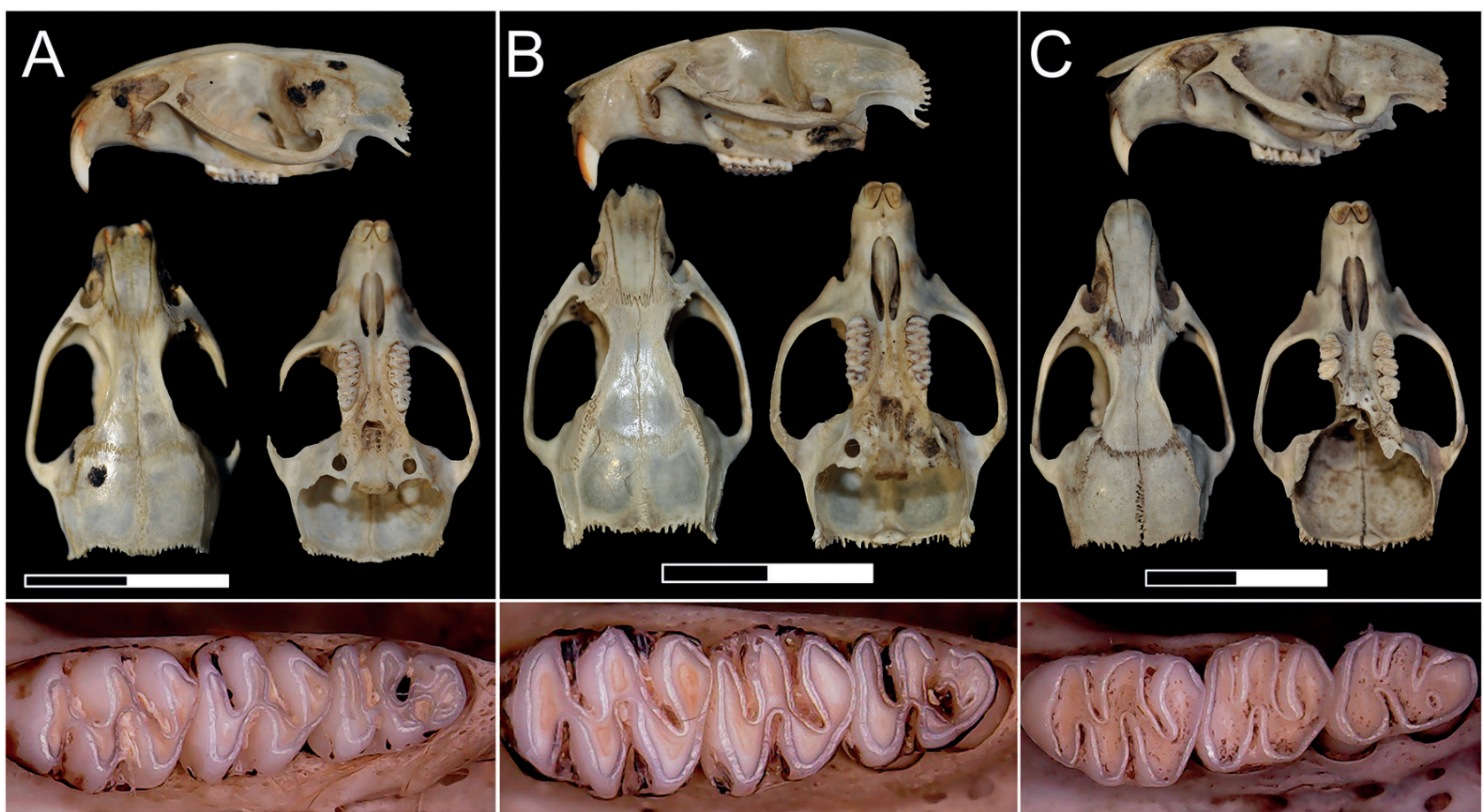

Figura 1. Restos craneanos de Holochilus chacarius hallados en distintas localidades de la Cuenca del Río Carcarañá, en vistas lateral izquierda, dorsal, ventral, y detalle de la serie molar derecha. Los ejemplares mostrados corresponden a las localidades de 1, 6 y 9 de la Figura 2. Es decir, A, individuo de Casilda; B, individuo de Los Quirquinchos; C, individuo de Montes de Oca 2. Escalas: 20 mm. 
Tabla 1. Localidades muestreadas en el sur de las provincias de Santa Fe. Se indican, en cada caso, el número de individuos (n) de Holochilus chacarius y el tipo de ambiente en el cual se obtuvieron los registros. Referencias: RU: Rural antropizado; RNAN: Rural no antropizado o natural; U: Urbano.

\begin{tabular}{|c|c|c|c|c|c|}
\hline \multirow{2}{*}{ Localidad } & \multirow{2}{*}{ Coordenadas } & \multirow{2}{*}{ Altitud (m) } & \multicolumn{2}{|r|}{$\mathbf{n}$} & \multirow[b]{2}{*}{$\mathbf{U}$} \\
\hline & & & RA & RNAN & \\
\hline 1- Casilda (departamento Caseros) & $32^{\circ} 55^{\prime} 02^{\prime \prime S}, 61^{\circ} 13^{\prime} 27^{\prime \prime} \mathrm{W}$ & 72 & - & 16 & - \\
\hline 3- Los Molinos 2 (departamento Caseros) & $33^{\circ} 03^{\prime} 38.7^{\prime \prime} \mathrm{S}, 61^{\circ} 20^{\prime} 21.09^{\prime \prime} \mathrm{W}$ & 82 & 1 & - & - \\
\hline 4- Arequito (departamento Caseros) & $33^{\circ} 04^{\prime} 02.84^{\prime \prime} \mathrm{S}, 61^{\circ} 27^{\prime} 56,60^{\prime \prime} \mathrm{W}$ & 78 & 2 & - & - \\
\hline 6- Los Quirquinchos (departamento Caseros) & $33^{\circ} 24^{\prime} 14.46^{\prime \prime} \mathrm{S}, 61^{\circ} 42^{\prime} 01.67^{\prime \prime} \mathrm{W}$ & 95 & 1 & - & - \\
\hline 7- Totoras (departamento Iriondo) & $32^{\circ} 35^{\prime} 43.26^{\prime \prime} \mathrm{S}, 61^{\circ} 13^{\prime} 54.02^{\prime \prime} \mathrm{W}$ & 39 & 5 & - & - \\
\hline 8- Montes de Oca 1 (departamento Belgrano) & $32^{\circ} 38^{\prime} 25.07^{\prime \prime} \mathrm{S}, 61^{\circ} 52^{\prime} 09.44^{\prime \prime} \mathrm{W}$ & 97 & - & 1 & - \\
\hline 9- Montes de Oca 2 (departamento Belgrano) & $32^{\circ} 35^{\prime} 06.81^{\prime \prime} \mathrm{S}, 61^{\circ} 49^{\prime} 06.88^{\prime \prime} \mathrm{W}$ & 94 & 1 & - & - \\
\hline
\end{tabular}

Los restos craneales fueron asignados a Holochilus chacarius dada la presencia de la siguiente combinación de caracteres: 1) mesolofo ausente en los molares superiores/ inferiores; 2) áreas de los proto e hipocono de contorno subrectangular (Hershkovitz 1955, Massoia 1976, Pardiñas \& Galliari 1998, Voglino et al. 2004, Pardiñas et al. 2013). Además, las medidas tomadas a los individuos (ver Tabla 2) se corresponden con las indicadas por otros autores para esta especie (Pardiñas et al. 2013, Brandao \& Nascimento 2015). Los ejemplares colectados en los distintos puntos de muestreo (ver Tabla 1) distan entre $50 \mathrm{Km}$ y $115 \mathrm{Km}$ al oeste del área de distribución conocida para la especie (ver Figura 2).

\section{Discusión}

Los registros aquí presentados extienden el rango de distribución de $H$. chacarius por hasta $115 \mathrm{Km}$ (Montes de Oca) hacia el oeste del río Paraná. Además, no se obtuvo ningún registro de $H$. vulpinus.
El río Paraná y sus grandes afluentes, como el Paraguay, son importantes "corredores" (es decir, rutas efectivas para la migración activa o pasiva) de la flora y fauna tropical hacia zonas templadas, donde pueden coexistir con especies locales (Ringuelet 1961). En el área estudiada existe un importante cuerpo de agua, el río Carcarañá, donde en sus riberas hay una mezcla de ambientes propios de pastizal pampeano (espartillares, ambientes halófitos, etc.), parcialmente modificados por actividad antrópica (mayormente áreas de cultivos y campos ganaderos), y ambientes boscosos típicos de la Ecorregión del Espinal. En este aspecto, $H$. chacarius y $H$. vulpinus han sido registrados en distintos sectores de la llanura Chaco-pampeana, a ambientes con alta diversidad de paisajes (Massa et al. 2014a), y ambas especies han sido halladas en egagrópilas de Tyto furcata, procedentes de agroecosistemas o cercanas a ellos (Scheibler \& Christoff 2004, Teta et al. 2010, Gonzalez-Fisher et al. 2012, 2017, Massa 2015).

Tabla 2. Medidas craneodentarias (en $\mathrm{mm}$ ) de los individuos de Holochilus chacarius mostrados en la Figura 1. M, media; DE, desvío estándar. Abreviaturas de las medidas (tomadas de Brandao \& Nascimento, 2015): LD, longitud del diastema; MTR, longitud de la serie molar; BM1, ancho del M1; LIF, longitud del foramen incisivo; BIF, ancho del foramen incisivo; BZP, ancho de la placa zigomática; LIB, ancho interorbitario.

\begin{tabular}{lcccc}
\hline Medidas & Ind. A & Ind. B & Ind. C & M \pm DE \\
\hline LD & 11.43 & 11.44 & 11.5 & $11.45 \pm 0.03$ \\
MTR & 7.18 & 7.17 & 7.23 & $7.19 \pm 0.03$ \\
BM1 & 2.14 & 2.14 & 2.16 & $2.14 \pm 0.01$ \\
LIF & 7.7 & 7.6 & 7.84 & $7.71 \pm 0.12$ \\
BIF & 2.51 & 2.52 & 2.57 & $2.53 \pm 0.03$ \\
BZP & 4.7 & 4.6 & 4.76 & $4.68 \pm 0.08$ \\
LIB & 4.6 & 4.6 & 4.8 & $4.66 \pm 0.11$ \\
Longitud del M1 & 2.84 & 2.86 & 2.92 & $2.87 \pm 0.04$ \\
Longitud del M2 & 1.81 & 1.82 & 1.9 & $1.84 \pm 0.04$ \\
Longitud del M3 & 2 & 2 & 2.06 & $2.02 \pm 0.03$ \\
Ancho del M1 & 2.14 & 2.15 & 2.16 & $2.15 \pm 0.01$ \\
Ancho del M2 & 2.1 & 2 & 2.1 & $2.06 \pm 0.05$ \\
Ancho del M3 & 1.72 & 1.72 & 1.74 & $1.72 \pm 0.01$ \\
\hline
\end{tabular}




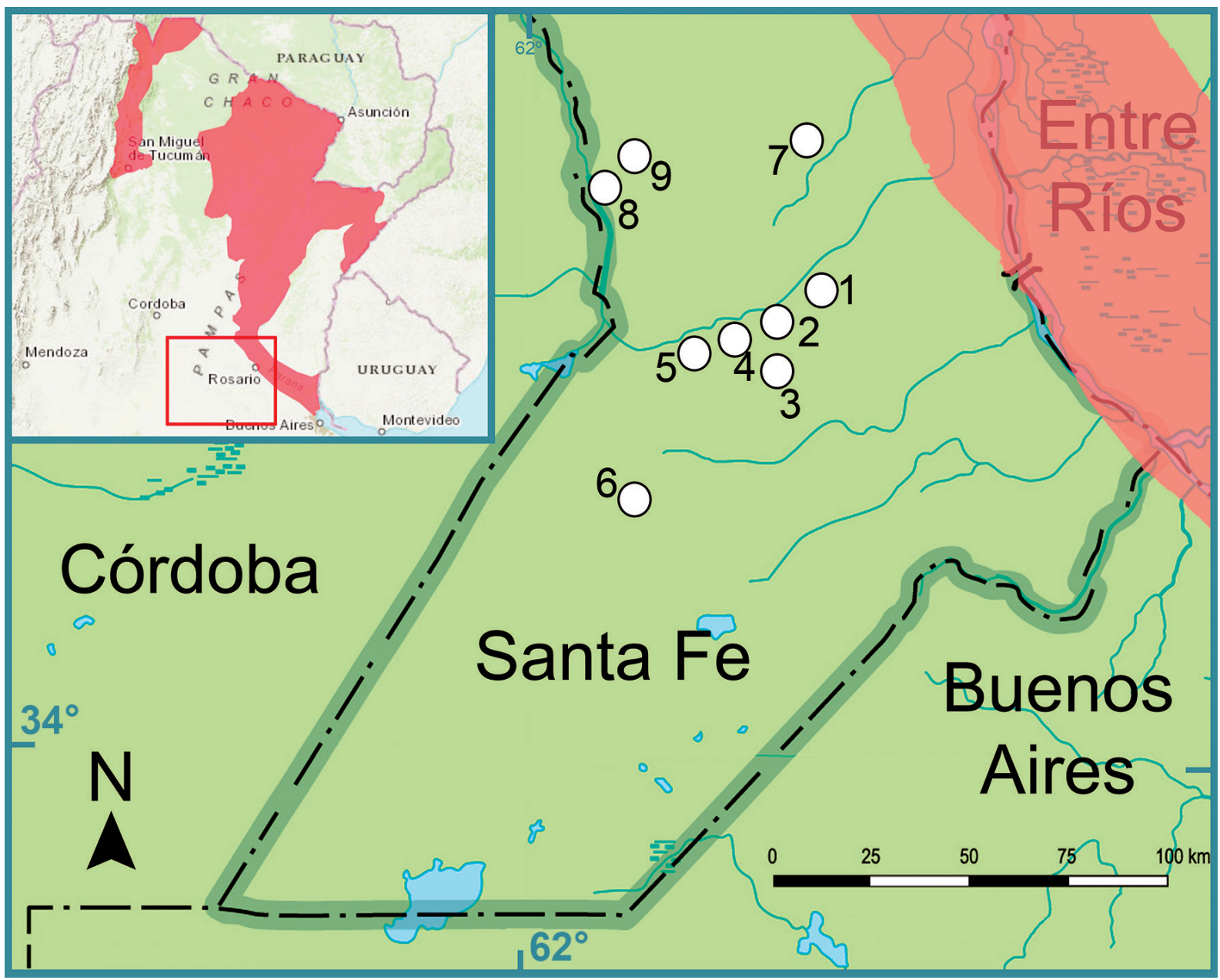

Figura 2. Área de estudio. En la esquina superior izquierda del recuadro se indica en rosado el área de distribución reconocida para la Holochilus chacarius en Argentina, según la última categorización nacional (Massa et al. 2019). El recuadro rojo indica el área de estudio. Los puntos numerados indican las localidades referenciadas en la Tabla 1.

Aunque el área de distribución de ambas especies es parcialmente simpátrica, son muy raras las referencias sobre hallazgos de ambos taxones en la misma localidad (Contreras et al. 2003, Voglino et al. 2004, Teta \& Pardiñas 2010, Massa et al. 2014a). Además, Prado et al. (2021) plantearon que las especies de Holochilus no sólo están separadas genéticamente, sino también ambientalmente, diferenciando a $H$. vulpinus como típicamente de pampas, mientras que $H$. chacarius sería más frecuentes en ambientes tipo pantanal. Por lo tanto, aunque en los mapas de distribución parecen ser especies simpátricas en distintos puntos de sus áreas de distribución, es probable que frecuenten diferentes ambientes. En los reportes de ambas especies en la misma localidad, en general $H$. vulpinus suele ser muy abundante, mientras que $H$. chacarius está poco representada (Chebez et al. 2005). Sólo en localidades del Chaco Húmedo se ha observado la tendencia contraria (Massoia 1971, 1976, Teta \& Pardiñas 2010, Udrizar Sauthier \& Carrera 2013), donde H. chacarius parece ser más abundante que $H$. vulpinus, y en pocas localidades se han reportado ambas especies en cantidades similares (Voglino et al. 2004). Por otro lado, son muy abundantes las citas de una de las dos especies, y la ausencia de la otra, particularmente cuando la muestra presenta una gran cantidad de individuos de una de las dos especies (Massoia et al. 1989, 1995, 1997, 1999, Teta \& Contreras 2003, Teta et al. 2006b, Massa et al. 2014a, Brandao \& Nascimento 2015).

Observando las áreas de distribución de H. vulpinus y $H$. chacarius, puede verse que esta última muestra una distribución por la zona chaqueña de Argentina, Paraguay, Bolivia, y parte del Cerrado de Brasil. Por otro lado, $H$. vulpinus parece habitar en un rango mayor de paisajes, ya que sus registros van desde el sur de la provincia de Buenos Aires, por el este de Argentina, hasta Paraguay y el sur de Brasil (Weksler et al. 2016a, 2016b). Esto es importante, porque según la última categorización de mamíferos de Argentina, H. vulpinus debería encontrarse en la zona centro y sur de la provincia de Santa Fe (Torres \& Massa 2019), mientras que H. chacarius llegaría hasta el delta inferior del Paraná en la provincia de Buenos Aires, a través de la cuenca del río Paraná (Massa et al. 2019). En los registros aquí presentados, se muestra que en el centro-sur de Santa $\mathrm{Fe}$, atravesando de oeste a este el ancho de la provincia habita $H$. chacarius, y no hay reportes concretos de $H$. vulpinus. 
Se ha planteado que $H$. chacarius y $H$. vulpinus habrían sufrido retracciones en sus áreas de distribución en las últimas décadas, posiblemente en asociación al cambio climático por actividad antrópica (Teta et al. 2014). Los nuevos hallazgos aquí reportados podrían deberse a la ausencia de muestreos detallados previos, como se ha sugerido para otras áreas donde se han reportado gran cantidad de ejemplares de $H$. chacarius (Voglino et al. 2004, Courtalon et al. 2013).

En conclusión, los registros que aquí se suman de $H$. chacarius, extienden su área de distribución en la provincia de Santa Fe, casi hasta el límite con la provincia de Córdoba. La intensificación de muestreos en áreas poco exploradas, con nuevas técnicas de muestreo y de identificación de taxones, ayudarán al mejor conocimiento de estos taxones y otros grupos con problemas similares.

\section{Literatura citada}

Andrews P. 1990. Owls, caves and fossils. British Museum Publications, London.

Bernard N, Michelat D, Raoul F, Quéré JP, Delattre P, Giraudoux P. 2010. Dietary response of Barn Owls (Tyto alba) to large variations in populations of common voles (Microtus arvalis) and European water voles (Arvicola terrestris). Canadian Journal of Zoology 88(4):416-426. https://doi.org/10.1139/Z10-011

Bilenca D, Codesido M, González-Fischer C, et al. 2012. Impactos de la transformación agropecuaria sobre la biodiversidad en la provincia de Buenos Aires. Revista del Museo Argentino de Ciencias Naturales, nueva serie, 14(2): 189-198.

Bó MS, Balandron AV, Biondi LM. 2007. Ecología trófica de Falconiformes y Strigiformes: tiempo de síntesis. Hornero 22: 97-115.

Bonvicino CR, Bezerra AM. 2003. Use of regurgitated pellets of barn owl (Tyto alba) for inventorying small mammals in the Cerrado of Central Brazil. Studies on Neotropical Fauna and Environment 38(1): 1-5

Brandão MV, Nascimento F. 2015. On the occurrence of Holochilus chacarius (Cricetidae: Sigmodontinae) in Brazil, with taxonomic notes on Holochilus species. Papéis Avulsos de Zoologia 55(3): 47-67. https://dx.doi. org/10.1590/0031-1049.2015.55.03

Cabrera A. 1976. Regiones fitogeográficas argentinas. Enciclopedia Argentina de Agricultura y Jardinería 2(1): 1-85.

Chebez JC, Pereira J, Massoia E, et al. 2005. Mamíferos de la Reserva el Bagual. En: Di Giacomo AG, Krapovickas SF, eds. Historia Natural y paisaje de la Reserva el Bagual, provincia de Formosa, Argentina. Temas de naturaleza y conservación - Monografía de Aves Argentinas № 4. Pp. 467-499.

Contreras JR, Teta P, Andrade A. 2003. Comentarios sobre el estatus de Calomys callosus (Rengger) y nuevos datos sobre la distribución de micromamíferos en el noroeste de la provincia de Corrientes. Revista del Museo Argentino de Ciencias Naturales, nueva serie 5: 73-78.

Coronel A, Sacchi 0. 2006. Climatología de eventos secos y húmedos en el Sur santafesino. Revista de Investigaciones de la Facultad de Ciencias Agrarias, Universidad Nacional de Rosario 9: 15-24.
Courtalon P, Lo Coco G, Bó R. 2013. Presencia de Holochilus chacarius Thomas, 1906 (Mammalia, Rodentia, Sigmodontinae) en el Delta Medio del río Paraná, Entre Ríos, Argentina. Revista del Museo Argentino de Ciencias Naturales, nueva serie 15(2): 289-293.

Errington PL. 1930. The pellet analysis method of raptor food habits study. The Condor 32(6): 292-296. https:// dx.doi.org/10.2307/1363377

Fulk GW. 1976. Owl predation and rodent martality: a case study. Mammalia 40: 423-427. https://doi.org/10.1515/ mamm.1976.40.3.423

Gómez-Villafañe IE, Miño M, Cavia R, et al. 2005. Guía de roedores de la provincia de Buenos Aires. Editorial L.O.L.A, Buenos Aires, Argentina. 100 pp.

González-Calderón A. 2017. Dieta de la lechuza de campanario (Tyto alba) en Ocoyoacac, Estado de México. Huitzil 18(2): 212-222. https://dx.doi.org/10.28947/ hrmo.2017.18.2.279

González-Fischer CM, Codesido M, Teta P, Bilenca D. 2012. Seasonal and geographic variation in the diet of Barn Owls (Tyto alba) in temperate agroecosystems of Argentina. Ornitología Neotropical 22(2): 295-305.

González Fischer CM, Cavia R, Picasso P, Bilenca D. 2017. Regional and local determinants of rodent assemblages in agroecosystems of the Argentine Pampas. Journal of Mammalogy 98(6): 1760-1767. https://dx.doi. org/10.1093/imammal/gyx121

Hershkovitz P. 1955. South American marsh rats genus Holochilus, with a summary of sigmodont rodents. Fieldiana, Zoology 37: 639-673.

Idoeta FM, Fernández FJ, de Santi NA, de Santi LJM. 2020. Quirópteros novedosos en egagrópilas de Tyto furcata (Strigiformes, Tytonidae) del Noreste Argentino. Historia Natural, tercera serie 10(3): 145-156.

Lewis JP, Collantes MB. 1974. La vegetación de la provincia de Santa Fe. Reseña general y enfoque del problema. Boletín de la Sociedad Argentina de Botánica 15: 343356.

Lyman RL. 2012. Rodent-prey content in long-term samples of barn owl (Tyto alba) pellets from the northwestern United States reflects local agricultural change. The American Midland Naturalist 167(1): 150-163. https://doi.org/10.1674/0003-0031-167.1.150

Marti C. 1987. Raptor food habits studies. In: BA Pendleton, BA Millsap, KW Cline and DM Bird, eds. Raptor management techniques manual. National Wildlife Federation, Washington DC. Pp. 67-79.

Martínez FF. 2010. Crónica de la soja en la región pampeana argentina. Para Mejorar la Producción (INTA, EEA Oliveros) 45: 141-146.

Massa C. 2015. Ecología del paisaje: comunidades de pequeños roedores de la Provincia de Entre Ríos. Tesis, Doctor en Ciencias Biológicas. Facultad de Ciencias Exactas y Naturales Universidad de Buenos Aires. Acceso 13/11/2021. http://hdl.handle.net/20.500.12110/ tesis n5808 Massa

Massa C, Teta P, Cueto GR. 2014a. Effects of regional context and landscape composition on diversity and composition of small rodent assemblages in Argentinian temperate grasslands and wetlands. Mammalia 78: 371-382. https://dx.doi.org/10.1515/mammalia-2013-0074

Massa C, Teta PV, Cueto GR. 2014b. Bat predation by Barn Owls (Tyto alba) in central-eastern Argentina. Chiroptera Neotropical 20(2): 1292-1296. 
Massa C, Gómez Villafañe I, d’Hiriart S. 2019. Holochilus chacarius. In: SAyDS-SAREM, eds. Categorización 2019 de los mamíferos de Argentina según su riesgo de extinción. Lista Roja de los mamíferos de Argentina. Acceso 13/11/2021. http://cma.sarem.org.ar

Massoia E. 1971. Caracteres y rasgos bioecológicos de Holochilus brasiliensis chacarius Thomas ("rata nutria") de la provincia de Formosa y comparaciones con Holochilus brasiliensis vulpinus (Brants) (Mammalia, Rodentia, Cricetidae). Revista de Investigaciones Agropecuarias, INTA, Serie 1, Biología y Producción Animal 8: $13-40$.

Massoia E. 1976. Mammalia. In: R Ringuelet, ed. Fauna de agua dulce de la República Argentina. Fundación Editorial Ciencia y Cultura: 1-128.

Massoia E. 1979. Descripción de un género y especie nuevos: Bibimys torresi (Mammalia-Rodentia-Cricetidae-Sigmodontinae-Scapteromyini). Physis 38: 1-7.

Massoia E. 1988. Presas de Tyto alba en Campo Ramón, Departamento de Oberá, Provincia de Misiones. Boletin APRONA 7: 4-16.

Massoia E, Fornes A. 1964. Pequeños mamíferos (Marsupialia, Chiroptera y Rodentia) y aves obtenidos en regurgitaciones de lechuzas (Strigiformes) del Delta bonaerense. Delta del Paraná, Investigaciones Agrícolas 4:27-34.

Massoia E, Tiranti SI, Torres MP. 1989. La depredación de pequeños mamíferos por Tyto alba en Canal 6, Delta bonaerense, partido de Campana, provincia de Buenos Aires. Boletin APRONA 13: 14-19.

Massoia E, Aprile G, Lartigau B. 1995. Análisis de regurgitados de Tyto alba de Estación Santa Margarita, departamento 9 de Julio, provincia de Santa Fe. Boletin APRONA 9(27): 19-21.

Massoia E, Heinonen de Fortabat S, Dieguez A. 1997. Análisis de componentes mastozoológicos y ornitológicos en regurgitados de Tyto alba de Estancia Guaycolec, Depto. Pilcomayo, Pcia. de Formosa, República Argentina. Boletin APRONA 32: 12-17.

Massoia E, Pastore H, Heinonen S. 1999. Análisis de regurgitados de Tyto alba de Escuela Pcial. № 17 "J. Sabiaur", Dto. Bermejo, Pcia. de Chaco. Boletin APRONA 36: 2-4.

Millán de la Peña N, Butet A, Delettre Y, et al. 2003. Response of the small mammal community to changes in western French agricultural landscapes. Landscape Ecology 18(3): 265-278. https://dx.doi. org/10.1023/A:1024452930326

Muñoz-Pedreros A, Rau J, Yañez J, eds). 2004. Aves Rapaces de Chile. ISBN: 9567279-08-X. Editorial CEA. 387 pp.

Pardiñas UF, Galliari CA. 1998. La distribución del ratón topo Notiomys edwardsii (Mammalia: Muridae). Neotrópica 44: 123-124

Pardiñas UFJ, Teta P, Cirignoli S, Podestá D. 2003. Micromamíferos (Didelphimorphia y Rodentia) de norpatagonia extra andina, Argentina: taxonomía alfa y biogeografía. Mastozoología Neotropical 10: 69-113.

Pardiñas UF, Teta P, Voglino D, Fernández FJ. 2013. Enlarging rodent diversity in west-central Argentina: a new species of the genus Holochilus (Cricetidae, Sigmodontinae). Journal of Mammalogy 94(1): 231-240. https:// doi.org/10.1644/12-MAMM-A-216

Patton JL, Pardiñas UF, D’Elía G, eds. 2015. Mammals of South America, volume 2: rodents. The University of Chicago Press, Chicago, IL, USA. xxvi + 1336 pp.
Podestá DH, Cirignoli S, Pardiñas UF. 2000. Nuevos datos sobre la distribución de Octodon bridgesii (Mammalia: Rodentia) en la Argentina. Neotrópica 46: 75-77.

Prado JR do, Knowles LL, Percequillo AR. 2021. New species boundaries and the diversification history of marsh rat taxa clarify historical connections among ecologically and geographically distinct wetlands of South America. Molecular Phylogenetics and Evolution. 155:106992. https://doi.org/10.1016/j.ympev.2020.106992

Rimoldi, P.G., Curti, M.G. 2021. Ecología trófica de la lechuza de campanario (Tyto furcata) en cuatro ambientes del sur de la provincia de Santa Fe, Argentina. Boletín del Museo Nacional de Historia Natural de Paraguay 25(1): 20-32.

Ringuelet RA. 1961. Rasgos fundamentales de la zoogeografía de la Argentina. Physis 22(63): 151-170.

Scheibler DR, Christoff AU. 2004. Small mammals in the diet of Barn Owls (Tyto alba) in agroecosystems of southern Brazil. Ornitología Neotropical 15(1): 65-70.

Teta P, Contreras JR. 2003. Primeros antecedentes de la dieta de la Lechuza de Campanario (Tyto alba) en el departamento Ñeembucú (Paraguay). Hornero 18(1): 57-59.

Teta P, Pardiñas UF. 2007. Mammalia, Didelphimorphia, Didelphidae, Chacodelphys formosa (Shamel, 1930): Range extension. Check List 3(4): 333-335.

Teta P, Pardiñas UF. 2010. Mammalia, Didelphimorphia and Rodentia, central Santa Fe Province, Argentina. Check List 6(4): 552-554.

Teta P, Pardiñas UF, D’Elía G. 2006a. Rediscovery of Chacodelphys: a South American marsupial genus previously known from a single specimen. Mammalian Biology 71(5): 309-314. https://doi.org/10.1016/i. mambio.2006.03.004

Teta P, Malzof S, Quintana R, Pereira J. 2006b. Presas del ñacurutú (Bubo virginianus) en el bajo delta del río Paraná (Buenos Aires, Argentina). Ornitología Neotropical 17(2): 441-444.

Teta P, González-Fischer CM, Codesido M, Bilenca DN. 2010. A contribution from Barn Owl pellets analysis to known micromammalian distributions in Buenos Aires province, Argentina. Mammalia 74: 97-103. https:// dx.doi.org/10.1515/mamm.2009.069

Teta P, Formoso A, Tammone M, et al. 2014. Micromamíferos, cambio climático e impacto antrópico: ¿Cuánto han cambiado las comunidades del sur de América del Sur en los últimos 500 años? Therya 5: 7-27. https:// dx,doi.org/10.12933/therya-14-183

Torres J, Massa C. 2019. Holochilus vulpinus. En: SAyDS-SAREM, eds. Categorización 2019 de los mamíferos de Argentina según su riesgo de extinción. Lista Roja de los mamíferos de Argentina. Acceso 13/11/2021 http://cma.sarem.org.ar

Trejo A, Lambertucci S. 2007. Feeding habits of Barn Owls along a vegetative gradient in northern Patagonia. Journal of Raptor Research 41(4): 277-287. https:// dx.doi.org/10.3356/0892-1016(2007)41[277:FHOB $\underline{\mathrm{OA}] 2.0 . \mathrm{CO} ; 2}$

Taylor I. 2004. Barn Owls: Predator-Prey Relationships and Conservation. Cambridge University Press, UK. 324 pp.

Udrizar-Sauthier DE, Carrera MG. 2013. Nuevos registros de micromamíferos (Rodentia, Chiroptera y Didelphimorphia) del Chaco Oriental, Argentina. Historia Natural, tercera serie 3(2): 51-60. 
Voglino D, Pardiñas UFJ, Teta P. 2004. Holochilus chacarius chacarius (Rodentia, Cricetidae) en la provincia de Buenos Aires, Argentina. Mastozoología Neotropical 11: 243-247.

Weksler M, Queirolo D, Brito D, et al. 2016a. Holochilus brasiliensis (errata version published in 2017). The IUCN Red List of Threatened Species 2016: e.T10217A115095907. Access 02/05/2021. https://dx.doi.org/10.2305/IUCN.UK.2016-3.RLTS. T10217A22345213.en

Weksler M, Brito D, Pardinas U, etal. 2016b. Holochilus chacarius (errata version published in 2017). The IUCN Red List of Threatened Species 2016: e.T10218A115096042. Access 02/05/2021. https://dx.doi.org/10.2305/ IUCN.UK.2016-3.RLTS.T10218A22345321.en

\section{Agradecimientos / Acknowledgments:}

Nuestro agradecimiento especial al Dr. Pablo Teta (Museo Argentino de Ciencias Naturales) por su ayuda en la identificación taxonómica de los individuos publicados. Además, uno de los autores (PGR) desea agradecer especialmente a Daniel Paiz y Cristian Alesio, por su ayuda en la colecta de las egagrópilas y en la identificación de los individuos.

\section{Conflicto de intereses / Competing interests:}

Los autores no incurren en conflictos de intereses.

\section{Rol de los autores / Authors Roles:}

PGR: Conceptualización, Investigación, Escritura - Preparación del borrador original, Redacción: revisión y edición; NRC: Investigación, Escritura- Preparación del borrador original, Redacción: revisión y edición.

\section{Fuentes de financiamiento / Funding:}

Esta investigación no recibió ninguna subvención específica de ninguna agencia de financiación.

\section{Aspectos éticos / legales; Ethics / legals:}

Los autores declaran que no violaron ni omitieron normas éticas o legales en esta investigación. No se buscó la aprobación ética para el presente estudio porque no involucro trabajo con animales vivos o un posible daño al ambiente. 